\title{
EMPRESA AGRARIA ASOCIATIVA: ANÁLISIS LEGISLATIVO DE LA SOCIEDAD AGRARIA DE TRANSFORMACIÓN VERSUS LA COOPERATIVA AGRARIA
}

\author{
Beatriz Llopis Gilabert \\ Economista y doctora \\ https://orcid.org/0000-0001-6719-3554
}

\section{RESUMEN}

La Sociedad Agraria de Transformación y la Cooperativa agraria se incluyen dentro de la categoría de empresas agrarias asociativas, que gozan de personalidad jurídica independiente de la de sus socios, que se dedican a la actividad agraria y se consideran fundamentales para el desarrollo del asociacionismo agrario. Además, son las fórmulas asociativas de las que se ha hecho uso el sector agrario. En el presente artículo una vez se han puesto de manifiesto unas consideraciones previas sobre la empresa agraria, se realizará un análisis legislativo de la Sociedad Agraria de Transformación y la Cooperativa agraria, desgranando las principales diferencias entre ambas sociedades relativas a el objeto social, la constitución, la forma jurídica, los límites de los socios, el reparto de beneficios y los órganos de gestión entre otros.

PALABRAS CLAVE: SAT, empresario agrario, Cooperativa agraria, órganos de gestión, personalidad jurídica, capital, socio.

CLAVES ECONLIT / ECONLIT DESCRIPTORS: P13, Q00, J54, M20.

Cómo citar este artículo/How to cite this article: LLOPIS GILABERT, B.: "Empresa agraria asociativa: análisis legislativo de la sociedad agraria de transformación versus la cooperativa agraria", CIRIEC-España, Revista Jurídica de Economía Social y Cooperativa, nº 39, 2021, pp. 165-197. DOI: 10.7203/CIRIEC-JUR.39.21445 


\section{AGRICULTURAL ASSOCIATIVE ENTITY: LEGISLATIVE ANALYSIS OF THE AGRICULTURAL PROCESSING COMPANY VERSUS THE AGRICULTURAL COOPERATIVE}

\section{EXPANDED ABSTRACT}

The agricultural company is distinguished from the commercial company basically by the requirement of professionalism demanded of the owner of the farm, the agricultural entrepreneur, as opposed to the owner of the commercial company, "the trader". This professionalism is required because the development of agricultural activity represents a state policy due to its social function and is therefore subject to a series of incentives and subsidies within the framework of the Community Agricultural Policy, and professionalism is therefore considered necessary in order to be eligible for public funds.

In addition, another differential element between farmer and trader is based on the risk assumed by a farmer, such as agricultural risk and the limitations of the biological cycle. This fact determines a difference between agricultural and commercial activity.

In principle, agricultural companies are civil (their legal regime will be adjusted in accordance with the provisions of the Civil Code), unless they adopt properly mercantile forms of incorporation such as the Limited Liability Company or the Public Limited Company, although the corporate purpose is not mercantile but civil in nature.

The subjective element of the agricultural company is constituted by the owner of the farm, who is considered in Article 2 of the LMEA as the natural or legal person who carries out the agricultural activity, organizing the assets and rights making up the farm with business criteria, and assuming the risks and the civil, social and tax liability that may arise from the management of the farm.

From the point of view of the owner of the farm business, the farm business is distinguished on the basis of two criteria. The first of these refers to the legal structure of the company. On the basis of this criterion, both the the agricultural processing company and the agricultural cooperatives are included in the category of associative agricultural companies. Therefore, they have legal personality independently of their partners and their corporate purpose is linked to agricultural activity.

It should be noted that both the the agricultural processing company and the agricultural cooperative constitute the traditional and widely accepted form of agricultural associationism, therefore, the interest of this article lies in the importance of making a legislative compari- 
son, explaining the main differences between both companies with the aim of being relevant for the choice of the ideal legal form for the agricultural entrepreneur.

It should be noted that while the the agricultural processing company is considered to be a civil company because this is defined in Law 1776/81, the agricultural cooperative has a "special" commercial purpose due to its complex management structure, unlike the commercial companies themselves.

Regarding the corporate purpose, in the The agricultural processing companys it is considered much broader, since it includes the production, marketing and transformation of agricultural, livestock or forestry products, among others, while in the agricultural cooperatives the main function is marketing.

As regards incorporation, both the the agricultural processing company and the agricultural cooperatives must be registered in their special registries in order to acquire legal personality, specifically in the general the agricultural processing company registry and the cooperative registry. however, in both companies a different percentage of paid-in capital is required in order to incorporate the company.

In the case of the the agricultural processing companys, an initial payment of at least $25 \%$ of the capital stock is required, and in the case of the agricultural cooperatives, the total amount of the paid-up contributions cannot be less than the minimum capital stock established in the bylaws, which means that it must be fully paid up.

Both natural persons and legal entities may be members of both agricultural cooperatives and agricultural cooperatives. In the case of natural person members, they can be owners of agricultural holdings, but also agricultural workers in the the agricultural processing companys; however, in the case of legal person members in the agricultural cooperatives, in addition to legal persons whose corporate purpose has agricultural purposes, the the agricultural processing companys themselves, and communities of property are also included. in addition, a maximum contribution limitation is established for a single member, which in the case of the the agricultural processing company is different if it is a natural person or a legal entity, and in the case of the agricultural cooperatives it is the same limit in the first degree cooperatives, whether they are legal entities or not.

The procedure for the acquisition, transfer and cancellation of membership in both the agricultural processing companys and the agricultural cooperatives is determined by what is established in the bylaws. However, the the agricultural processing companys are much more open and less restrictive than the cooperatives as regards the transfer procedure, since it 
is not limited to having the status of members beforehand, nor is it necessary to acquire it within a certain period of time, as occurs in the case of the agricultural cooperative.

Focusing on the liability and distribution of profits of the members, while in the the agricultural processing company the members are liable to third parties in an unlimited manner with their assets and the distribution of profits is proportional to the contribution to the capital of each member or according to the bylaws, in the cooperatives the liability for social debts is limited to the contribution to the capital stock and the distribution of profits is proportional to the operations, activities or services developed by the members in the cooperative.

The process of dissolution and liquidation of the company is much more defined and detailed in the the agricultural processing companys than in the cooperatives, since once the dissolution of the company has been approved, the liquidation phase of the company begins in both companies, which will be limited in the case of the the agricultural processing companys to one year and with an odd number of liquidators, but with a maximum limit of five, while in the agricultural cooperatives neither the maximum term of the liquidation phase nor the maximum number of liquidators is specified, although they must also be an odd number.

It should be noted that the regime, control and organization of cooperatives in general and of agricultural cooperatives in particular is much stricter than that of other types of agricultural companies such as the agricultural processing companys, because it imposes an intervention as a control body with a supervisory role.

Finally, in tax matters, the agricultural processing companys and agricultural cooperatives present important differences in direct taxation. in particular, agricultural cooperatives enjoy a special rebate of $95 \%$ of the quota and surcharges on rural property and are taxed at a special corporate tax rate of $20 \%$, while the the agricultural processing companys do not enjoy any ibi rebate and are taxed at the general corporate tax rate of $25 \%$.

KEYWORDS: ATC, the owner of the farm, The agricultural cooperative, board of directors, legal form, capital, parther. 


\section{SUMARIO}

1. Consideraciones previas y metodología. 2. Empresa agraria asociativa. 3. Principales diferencias entre Sociedad Agraria de Transformación (SAT) y Cooperativa Agraria. 3.1. Concepto, objeto social y forma jurídica. 3.2. Constitución y capital social. 3.3. Número, tipos y limitaciones de los socios. 3.4. Procedimiento de adquisición, transmisión y pérdida de la condición de socios. 3.5. Responsabilidad de los socios y reparto de beneficios de la sociedad. 3.6. Extinción de la sociedad. 3.7. Órganos de gestión. 3.8. Fiscalidad 4. Conclusiones. Bibliografía.

\section{Consideraciones previas y metodología}

En primer lugar, es necesario acudir al concepto de empresa agraria que viene definido por la Ley 19/1995 de 4 de julio de Modernización de Explotaciones Agraria (en adelante, LMEA) como el "conjunto de bienes y derechos organizados empresarialmente por su titular para el ejercicio de la actividad agraria, primordialmente con fines de mercado y que constituye en sí misma una unidad técnico-económica”.

El elemento subjetivo de la empresa agraria, lo constituye el titular de la explotación agraria que se considera en el artículo segundo de la LMEA como la "persona física o jurídica que ejerce la actividad agraria organizando los bienes y derechos integrantes de la explotación con criterios empresariales, y asumiendo los riesgos y la responsabilidad civil, social y fiscal que puede derivarse de la gestión de la explotación”.

En virtud de esta definición del titular de la explotación agraria, los requisitos que conforman a la empresa agraria se organizan a partir del estudio de cuatro conceptos (Millán Salas, 1993:215-226):

En primer lugar, el empresario agrario ha de desempeńar la actividad agraria de acuerdo con el principio de Economicidad. Este principio consiste en alcanzar el máximo beneficio en la explotación agraria utilizando la mínima dotación de recursos posible.

El segundo requisito hace referencia a la Organicidad. En particular, el empresario agrario debe organizar los elementos materiales, inmateriales, objetivos, y subjetivos que intervienen en la producción de bienes y servicios y sus fases complementarias. Estos elementos son de muy diversa naturaleza, pues varían en función de la tradición del cultivo, el clima y las condiciones geológicas de la región.

El tercer requisito que debe exigirse al empresario para tener tal consideración, se refiere la Profesionalidad. En este sentido, el desarrollo de la actividad agraria repre- 
senta una política de estado por su función social y por ello, está sometida a una serie de estímulos y subvenciones en el marco de la Política Agraria Comunitaria y se exige la profesionalidad para poder optar a ayudas públicas.

En cambio, en el caso de la activad comercial no existe esta protección ni fondos públicos para su incentivación, por lo que no exige dicha profesionalidad al comerciante (Carrozza \& Zeledón Zeledón, 1990:207-212).

Además, otro elemento diferencial entre agricultor y comerciante se basa en el riesgo asumido por un agricultor como son el riesgo agrícola y las limitaciones del ciclo biológico. Este hecho determina una diferencia entre actividad agraria y mercantil.

En principio, las empresas agrarias son civiles (su régimen jurídico se ajustará conforme a lo dispuesto en el Código civil), salvo que adopten formas de constitución propiamente mercantiles como la Sociedad de Responsabilidad Limitada o la Sociedad Anónima, aunque el objeto social no tenga carácter mercantil sino civil (Herrera Campos, 2005:67-70).

En cuarto lugar, para alcanzar la condición de empresario agrario el titular debe asumir el requisito de imputabilidad. Se trata de un concepto jurídico que atribuye al sujeto las consecuencias de su obrar. La imputabilidad hace referencia a la asunción de riesgos derivados de la gestión de una actividad empresarial por parte del empresario agrícola, que podría trasladar a terceras personas la asunción económica del riesgo, sin que ello implique que quede exento de responsabilidad. Como argumenta Millán (1993:220) se considera muy ligada a la economicidad. Si la actividad empresarial es económica el empresario tiene que responder de la misma.

Dicho requisito también se recoge en la definición legal descrita anteriormente de la LMEA, referente al titular de la explotación agraria, cuando afirma que "el titular de la explotación agraria asumirá los riesgos y responsabilidades civil, social y fiscal que puedan derivarse de la gestión de la explotación”.

Por otro lado, desde el punto de vista del sujeto titular de la empresa agraria, las empresas agrarias se clasifican en base a dos criterios principales: el primero de ellos, hace referencia a la estructura jurídica de la empresa, o lo que es lo mismo, a la personalidad civil, que responde a la facultad de ser sujeto de derechos y obligaciones; y el segundo requisito hace referencia al régimen de tenencia de la tierra o al tipo de derecho personal o real que habilita al agricultor para el ejercicio de la titularidad empresarial agraria.

Dentro de la primera clasificación de empresas agrarias en base a la estructura jurídica de la empresa, distinguimos a su vez dos tipos de empresarios:

En primer lugar, el empresario agrario individual, que se define como una persona física que puede ejercer la actividad agraria mediante un régimen de titularidad 
individual; un régimen de titularidad compartido, en caso de existir un matrimonio o pareja de hecho (análoga relación de afectividad); o bien a través de una comunidad de bienes o sociedad civil sin personalidad jurídica.

$\mathrm{Y}$, en segundo lugar, el empresario agrario asociativo, que goza de personalidad jurídica independiente de la de sus socios y que se dedican a la actividad agraria.

Cabe destacar, que en el presente artículo se pretende realizar una comparativa jurídica basada en la legislación vigente entre las dos fórmulas asociativas más importantes en el sector agrario español, siendo estas las Cooperativas agrarias y las Sociedades Agrarias de Transformación (en adelante, SAT), describiendo sus principales características. Se ha utilizado para ello una metodología basada en la investigación documental, que se caracteriza por la observación de fenómenos a analizar cómo son las fuentes o documentos escritos y que, en nuestro caso concreto, son los textos jurídicos que poseen carácter legal y son fuente del derecho.

Por tanto, el principal método de investigación utilizado en el trabajo ha sido el lingüístico normativo que resulta fundamental para el estudio y análisis de la norma y del discurso jurídico y, en particular, se ha analizado la normativa legal en materia agraria como LMEA, así como se ha profundizado en el estudio del Real Decreto 1776/81 que regula las SAT y la Ley de Cooperativas 27/1999 que regula las Cooperativas.

\section{Empresa agraria asociativa}

Siguiendo la clasificación anterior de la tipología de empresas agrarias en base al sujeto titular de la explotación, cuando el empresario agrario o titular de la explotación agraria es una persona jurídica, estamos ante empresas agrarias de base asociativa que gozan de personalidad jurídica independiente de la de sus socios y que se dedican a la actividad agraria.

Ya en la década de los ochenta del siglo pasado, el profesor Vattier (1978b:1.582) se refería al complejo instituto de la empresa agraria colectiva cuya titularidad corresponde a un sujeto de tipo pluripersonal y que se organiza en base a un esquema típico de contrato de sociedad con la finalidad de desarrollar en común una actividad agraria.

Procedemos a analizar si la empresa agraria en modalidad asociativa cumple con los requisitos generales de la empresa agraria definidos con anterioridad, tales como;

- En primer lugar, el requisito de economicidad debe estar presente en todas las personas que intervengan como titulares de esta, al igual que, todas las personas 
que intervengan han de contribuir a la formación y organización de la explotación agraria común.

- En segundo lugar, el requisito de profesionalidad se considera un requisito complejo en las empresas societarias, ya que cuando la empresa agraria adopta la estructura societaria, la profesionalidad hace referencia a un atributo que debe exigirse a la persona jurídica que resulte del contrato de sociedad y no al conjunto de socios que la constituyan.

- En tercer lugar, el requisito de imputabilidad. Ha de ser también conjunta cualesquiera que sean los acuerdos internos de los socios en orden a la asunción de riesgos y al reparto de las eventuales ganancias o pérdidas debiendo responder ante terceros según la estructura típica que tenga la empresa colectiva.

- Y, por último, los requisitos relativos a la actividad económica de la empresa agraria. La empresa agraria debe quedar comprendida dentro de los límites de la actividad agraria que incluye, además de las actividades agrarias primarias de producción agrícola, las actividades de transformación y de comercialización en las que adopta una estructura societaria de tipo mercantil o de tipo cooperativo.

Además, las personas jurídicas pueden adquirir y poseer bienes de toda clase, así como contraer obligaciones y ejercitar acciones civiles o criminales conforme a las leyes y a las reglas de su constitución. Por tanto, desde el punto de vista de las relaciones internas, son patrimonios separados el patrimonio de los socios y el patrimonio de la sociedad y, desde el punto de vista externo de relaciones frente a terceros, existe un tipo de responsabilidad más limitada a las aportaciones que realicen a la sociedad.

Una de las ventajas de la existencia de las distintas formas jurídicas de estructura asociativa, según Carretero (2003:116), se basa en la posibilidad que tienen los agricultores de poner recursos en común con el objeto de crear explotaciones agrarias más rentables y hacer frente a las dificultades características del sector agrario como factores climáticos, cambios en la PAC o ciclos económicos, entre otros.

Cabe acudir al artículo 35 del Código Civil para buscar la definición de personas jurídicas. Según dicho artículo, se incluyen como personas jurídicas:

1. Las asociaciones de interés particular sean civiles, mercantiles o industriales a las que la ley conceda personalidad propia independiente de la de cada uno de los asociados. Se engloban dentro de este grupo las SAT y la Cooperativa agraria.

2. Las corporaciones, asociaciones y fundaciones de interés público reconocidas por la ley. Su personalidad empieza desde el instante mismo en que con arreglo a derecho hubiesen quedado válidamente constituidas. 


\section{Principales diferencias entre Sociedad Agraria de Transformación (SAT) y Cooperativa Agraria}

La SAT y la cooperativa agraria son empresas agrarias asociativas que se consideran fundamentales para el desarrollo del asociacionismo agrario. Por tanto, tienen personalidad jurídica independientemente de sus y socios y su objeto social se vincula a la actividad agraria.

En la página siguiente, presentamos una tabla resumen en la que establecen las principales diferencias entre ambas empresas agrarias asociativas que vamos a ir desgranando a lo lardo del presente artículo con el objetivo de analizar de forma más exhaustiva dichas formas jurídicas, que se consideran las fórmulas asociativas de las que se ha hecho uso el sector agrario español.

\subsection{Concepto, objeto social y forma jurídica}

En primer lugar, conviene destacar que se sitúa el antecedente inmediato de las SAT en los antiguos Grupos Sindicales de Colonización, creados por Orden del Ministerio de Agricultura de 11 de junio de 1941 en el seno de las Hermandades Sindicales o de los Sindicatos Locales de la Falange Tradicionalista de las J.O.N.S (Cuenca Anaya, 1983:49).

En la actualidad, las SAT se rigen fundamentalmente por el Real Decreto 1776/81 por el que se aprueba el Estatuto que regula las SAT y por la Orden de 14 de septiembre de 1982, que desarrolla dicho Real Decreto, así como y subsidiariamente por las normas que resulten aplicables a las sociedades civiles.

En este sentido, en el artículo 1 del Real Decreto 1776/81 se definen las SAT como "sociedades civiles de finalidad económico-social, constituidas para la producción, comercialización y transformación de productos agrarios, ganaderos o forestales, la realización de mejoras en el medio rural, la promoción y el desarrollo agrarios y la prestación de servicios comunes que sirvan a aquella finalidad".

El autor Beltrán (1997:79) plantea dos cuestiones en torno a dicha definición con el objetivo de establecer la verdadera esencia de este tipo de sociedad. La primera cuestión, hace referencia a la SAT como sociedad, puesto que a las sociedades se les exige que tengan ánimo de lucro en su actividad. Cabe destacar que, esto parece claramente oponerse a la finalidad que persigue la SAT, una finalidad económico-social propia de entidades que no persiguen ánimo de lucro en su actividad. 


\begin{tabular}{|c|c|c|}
\hline & SAT & Cooperativa Agraria \\
\hline Regulación & RD 1776/81 SAT & LC $27 / 1999$ \\
\hline Objeto social & $\begin{array}{l}\text { - Producción } \\
\text { - Comercialización } \\
\text { - Transformación }\end{array}$ & - Comercialización \\
\hline Forma jurídica & - Sociedad civil & - Sociedad mercantil \\
\hline Constitución & - Inscripción Registro SAT & - Escritura pública. Registro Cooperativa \\
\hline Capital Social & - $>25 \%$ desembolsado & - $100 \%$ desembolsado \\
\hline N.o socios mínimos & $\cdot 3$ & $\begin{array}{l}\text { - Cooperativas } 1^{\circ} \text { Grado: } 3 \text { socios } \\
\text { - Cooperativas } 2{ }^{\circ} \text { Grado: } 2 \text { socios }\end{array}$ \\
\hline Tipo de socios & $\begin{array}{l}\text { - Personas físicas: } \\
\text { - Titulares de explotaciones agrarias. } \\
\text { - Trabajadores agrarios } \\
\text { - Personas jurídicas: } \\
\text { - El objetivo social tenga fines agrarios }\end{array}$ & $\begin{array}{l}\text { - Personas físicas: } \\
\text { - Titulares de explotaciones agrícolas, ganaderas y } \\
\text { forestales } \\
\text { - Personas jurídicas: } \\
\text { - SAT } \\
\text { - Comunidad de Regantes } \\
\text { - Comunidad de bienes } \\
\text { - Comunidad de aguas } \\
\text { - Sociedades civiles o mercantiles con fines agrarios }\end{array}$ \\
\hline $\begin{array}{l}\text { Límite } \\
\text { aportaciones de un } \\
\text { socio }\end{array}$ & $\begin{array}{l}\text { - Personas físicas: }>1 / 3 \text { capital social } \\
\text { - Personas jurídicas: } 50 \% \text { capital }\end{array}$ & $\begin{array}{l}\text { - Cooperativas } 1^{\circ} \text { Grado: }>1 / 3 \text { capital } \\
\text { - Socios colaboradores: } 45 \% \text { capital }\end{array}$ \\
\hline $\begin{array}{l}\text { Procedimiento alta } \\
\text { y baja socios. }\end{array}$ & $\begin{array}{l}\text { - Alta: Estatutos } \\
\text { - Transmisión: } \\
\text { - Actos inter vivos: No limitada } \\
\text { - Mortis causa: No ser previamente socios } \\
\text { - Baja: } \\
\text { - baja voluntaria } \\
\text { - exclusión forzosa }\end{array}$ & $\begin{array}{l}\text { - Alta: Estatutos } \\
\text { - Transmisión: } \\
\text { - Actos inter vivos: Si Limitada } \\
\text { - Mortis causa: Ser socios previamente } \\
\text { - Baja: } \\
\text {-baja voluntaria } \\
\text { - baja forzosa } \\
\text { Expulsión }\end{array}$ \\
\hline Responsabilidad & $\begin{array}{l}\text { - Ilimitada } \\
\text { - Personal } \\
\text { - Mancomunada }\end{array}$ & - Limitada \\
\hline Reparto Beneficios & - Proporcional al capital & - Proporcional a la actividad \\
\hline $\begin{array}{l}\text { Extinción de la } \\
\text { Sociedad }\end{array}$ & $\begin{array}{l}\text { - Causas disolución } \\
\text { - Acuerdo de disolución: } \\
\text { - } 1^{\text {a }} \text { convocatoria: }>2 / 3 \text { socios } \\
\text { - } \text { - }^{\text {a convocatoria }>1 / 2} \\
\text { - Cumplimiento del plazo de constitución } \\
\text { - La conclusión del objeto social o imposibilidad de } \\
\text { realizarlo } \\
\text { - La cesación o abandono de actividades } \\
\text { - La alteración sustancial de los caracteres propios } \\
\text { que configuran la SAT } \\
\text { - Inscripción disolución: } \\
\text { - Registro SAT } \\
\text { - Periodo liquidación } \\
\text { - Limitado } \\
\text { - No liquidadores: } \\
\text { - Número no superior } 5\end{array}$ & $\begin{array}{l}\text { - Causas disolución } \\
\text { - Acuerdo de disolución: >2/3 socios } \\
\text { - Cumplimiento del plazo de constitución } \\
\text { - La conclusión del objeto social o imposibilidad de } \\
\text { realizarlo } \\
\text { - La cesación o abandono de actividades } \\
\text { - La paralización de los órganos sociales } \\
\text { - Fusión, absorción o escisión. } \\
\text { - La reducción del número de socios por debajo del } \\
\text { capital social } \\
\text { - Inscripción disolución: } \\
\text { - Registro Cooperativas } \\
\text { - Periodo liquidación: } \\
\text { - No limitado } \\
\text { - No liquidadores: } \\
\text { - Número impar }\end{array}$ \\
\hline Órganos de gestión & $\begin{array}{l}\text { - Asamblea General } \\
\text { - Junta Rectora } \\
\text { - Presidente }\end{array}$ & $\begin{array}{l}\text { - Asamblea General } \\
\text { - Consejo Rector } \\
\text { - Intervención }\end{array}$ \\
\hline Fiscalidad & $\begin{array}{l}\text { - IBI } \\
\text { - No hay bonificación } \\
\text { - IS } \\
-25 \% \\
\text { - No hay bonificación }\end{array}$ & $\begin{array}{l}\text { - IBI } \\
\text { - Bonificación } 95 \% \\
\text { - IS } \\
\text { - } 20 \% \\
\text { - Bonificación } 50 \% \text { CI }\end{array}$ \\
\hline
\end{tabular}


No obstante, podemos concluir esta cuestión afirmando que la finalidad económico-social no es incompatible con el ánimo el lucro, puesto que a la luz lo dispuesto en el Real Decreto 1776/81 que establece en su artículo 7.d) que "los socios tendrán derecho a obtener beneficios comunes proporcionales a su participación" y, por tanto, queda patente el ánimo de lucro que se exige a las entidades societarias.

La segunda cuestión que se plantea en torno a la SAT hace referencia a la consideración de la SAT como sociedad civil. Se trata de una cuestión sumamente debatida y confusa, puesto que las SAT pueden desarrollar tanto actividades civiles como mercantiles. Las actividades que desarrollan las SAT enumeradas en el artículo 1 del Real Decreto 1776/81 son civiles, salvo la transformación y comercialización de productos que es mercantil. Pese a esta combinación de actividades, el Real Decreto le otorga la consideración de sociedad civil.

En caso de que las SAT tuvieran como objeto único la transformación y comercialización de productos agrarios, Beltrán (1997:98) considera que se rompería con la conexión de actividad agrícola y tendrían la consideración de sociedad mercantil.

Sin embargo, el Real Decreto 1776/81 no permite como objeto único la realización de actividades de transformación y comercialización de productos agrarios, sino que, dicho objeto debe ir unido a la producción de estos productos. Además, solo pueden ostentar la condición de socios las personas físicas o jurídicas que persigan fines agrarios. Por tanto, podemos afirmar con rotundidad resultando incuestionable que la SAT se considera una sociedad claramente civil.

En el caso de las cooperativas agrarias en España carecen de regulación particular. Hay que acudir, por tanto, a lo dispuesto en la Ley de Cooperativas 27/1999, en adelante LC 27/1999, que contiene el régimen jurídico de las cooperativas que desarrollen su actividad en el territorio de varias Comunidades Autónomas, salvo en el caso de Comunidades Autónomas que posean una Ley de Cooperativas propia.

No obstante, la mayor parte de la legislación autonómica se ha inspirado en la legislación estatal común, por tanto, vamos a centrarnos en las características de las Cooperativas reguladas en la ley estatal.

El artículo 1 de la LC 27/1999 define la cooperativa como "sociedad constituida por personas que se asocian, en régimen de libre adhesión y baja voluntaria para realizar actividades empresariales encaminadas a satisfacer sus necesidades y aspiraciones económicas y sociales, con estructura y funcionamiento democrático, conforme a los principios y formulados por la alianza cooperativa internacional, en los términos resultantes de la presente Ley".

Por tanto, de lo que se desprende de la voluntad del legislador es configurar una asociación autónoma de personas unidas voluntariamente, con el objetivo de desarrollar una actividad económica usando una empresa para ello y donde prevale el 
principio de ayuda mutua para alcanzar los objetivos generales de la empresa en su conjunto, pero también de los socios en particular.

Podemos clasificar las cooperativas en dos tipos. En primer lugar, las cooperativas de primer grado, que pueden incluir tanto personas físicas como personas jurídicas, pero en ningún caso otras cooperativas y deberán estar integradas por al menos tres socios. Y las cooperativas de segundo grado, que estarán constituidas por un mínimo de dos cooperativas.

A su vez, las cooperativas de primer grado podrán clasificarse de la siguiente forma (art 6.1 LC 27/1999):

- Cooperativas de trabajo asociado.

- Cooperativas de consumidores y usuarios.

- Cooperativas de viviendas.

- Cooperativas agroalimentarias.

- Cooperativas de explotación comunitaria de la tierra.

- Cooperativas de servicios

- Cooperativas del mar.

- Cooperativas de transportistas.

- Cooperativas de seguros.

- Cooperativas sanitarias.

- Cooperativas de enseñanza.

- Cooperativas de crédito.

Y las cooperativas de segundo grado tendrán la clasificación anterior, siempre que todas las cooperativas socias pertenezcan a la misma clase añadiendo en tal caso la expresión de "segundo grado".

Siguiendo lo dispuesto en el artículo 93 de la LC 27/1999, "las cooperativas agrarias asocian a titulares de explotaciones agrícolas, ganaderas o forestales que tienen como objeto todo tipo de actividades y operaciones encaminadas al mejor aprovechamiento de las explotaciones de sus socios, de sus elementos o componentes de la cooperativa, a la mejora de la población agraria y del desarrollo del mundo rural, así como a atender cualquier otro fin o servicio propio de la actividad agraria, ganadera o forestal, o directamente relacionados con ellas, y con su implantación en el medio rural”.

Por tanto, en función de la definición anterior la cooperativa sea agraria o no, se configura como una sociedad mercantil "especial", puesto que se trata de la asociación de empresarios agrarios con el fin de desarrollar actividades económicas y empresariales principalmente de comercialización agraria y además, por su estructura 
compleja de gestión como veremos posteriormente, nos conduce a pensar que evoca a la propia empresa (Macías Ruano, 2017:55-58).

No obstante, tienen su propia regulación y no se inscriben en el registro mercantil como si lo hacen las sociedades mercantiles tradicionales de ahí que se catalogue como especial.

Y, además, el objeto social en el caso de cooperativas agroalimentarias deberá vincularse a la actividad agraria o al desarrollo rural, pero desarrollan principalmente actividades de comercialización agraria, a diferencia de las SAT que incluyen en el objeto social además la producción y transformación agraria.

Cabe mencionar como ejemplos de actividades susceptibles desarrolladas por las cooperativas agroalimentarias las siguientes:

a) Entre las actividades destinadas a mejorar al aprovechamiento de las explotaciones incluimos:

- Adquirir, elaborar o producir por cualquier procedimiento, para la cooperativa o para las explotaciones de sus socios, los elementos necesarios para la producción y el fomento agrario, alimentario y rural.

- Actividades de consumo y servicios para sus socios y demás miembros de su entorno social y territorial, fomentando actividades encaminadas a la mejora de la población agraria y el medio rural.

- Actividades necesarias que faciliten la mejora técnica, económica, laboral o ecológica de la cooperativa o de las explotaciones de los socios.

b) Entre las actividades agrarias típicas o directamente relacionadas con ellas encontramos:

- Conservar, manipular, transformar, transportar y distribuir, incluso directamente al consumidor, los productos procedentes de las explotaciones de la cooperativa y de sus socios en su estado natural o previamente transformados.

- Adquirir, parcelar, sanear y mejorar terrenos destinados a la agricultura, la ganadería o los bosques, así como la construcción y explotación de las obras e instalaciones necesarias a estos fines.

Dentro del sistema agroalimentario, el cooperativismo puede jugar un papel importante en la concentración de la oferta de producción agraria, con el fin de conseguir mejores condiciones y precios de venta en la negociación con grandes cadenas distribuidoras.

En este sentido, la cooperativa puede ostentar la titularidad de explotaciones agrarias propias y ejercer la actividad en su propio nombre, explotando tierras de su titularidad y contratando al personal necesario para que las trabaje o sean trabajadas por los socios. Se trata de explotación capaz de generar por sí misma pro- 
ducción agraria, trabajo y rentabilidad en beneficio de los socios y de su ámbito geográfico.

c) Las actividades encaminadas a la promoción y mejora de la población agraria y del medio rural.

En esta tercera modalidad, las actividades tienen como misión elevar el nivel de calidad de vida de la población agraria y rural.

\subsection{Constitución y capital social}

Las SAT gozarán de personalidad jurídica y plena capacidad de obrar para el cumplimiento de su finalidad desde su inscripción en el Registro General de las SAT, siendo su patrimonio independiente del de sus socios. El Registro General de las SAT, que será único, ajustará sus funciones a los principios de publicidad formal y material, legalidad y legitimación, conforme a las normas que se dicten al respecto.

En cuanto al capital social, será variable ya que no se establece un límite y está constituido por el valor de las aportaciones dinerarias o no realizadas por los socios en el acto de constitución o en virtud de posteriores acuerdos.

Los resguardos representativos del capital se configuran como "resguardos nominativos autorizados con las firmas del presidente y del secretario de la sociedad. Se materializarán en una parte alícuota del capital social, de forma que no ofrezca duda la aportación individual de cada socio. No tendrán el carácter de títulos valores y su transmisión no otorgará la condición del socio adquirente" (Artículo 8.1 del Real Decreto 1776/81).

Solo se podrá constituir la sociedad si al menos el $25 \%$ del capital social está totalmente suscrito y desembolsado. El resto se desembolsará según se determine, siempre dentro del plazo de seis años. Además, las aportaciones sociales podrán ser dinerarias o no dinerarias. En el caso de estas últimas, deberán valorarse en metálico y en una cifra no superior a lo que determinen las leyes fiscales en los procedimientos de comprobación de valores (artículo 8.4 del Real Decreto 1776/81).

En el caso de las cooperativas en general incluyendo las cooperativas agroalimentarias deberán ser inscritas en el Registro de Sociedades Cooperativas ${ }^{1}$ previsto en el Real Decreto 136/2002, de 1 de febrero. Dicha inscripción será obligatoria, pues con ella adquiere la cooperativa su personalidad jurídica y así la inscripción posee 
eficacia constitutiva. En la escritura pública además de otros contenidos, se incluirán los estatutos que hayan de regir el funcionamiento de la cooperativa ${ }^{2}$.

El capital social está constituido por aportaciones obligatorias y voluntarias de socios y asociados que podrán ser dinerarias o no si lo establecen los estatutos o lo acuerda la Asamblea General. Los Estatutos fijarán el capital social mínimo que deberá estar totalmente desembolsado, correspondiendo un $25 \%$ al menos, a las aportaciones obligatorias para adquirir la condición de socio. Asimismo, determinarán la forma y plazos para desembolsar el resto. Además, los estatutos podrán fijar si las aportaciones devengarán intereses en cuyo caso no podrá exceder en más de tres puntos del interés básico del Banco de España. (Art. 72 y 73 LC 27/1999).

En resumen, tanto la SAT como las cooperativas agrarias deberán inscribirse de forma obligatoria en sus registros especiales para adquirir la personalidad jurídica. No obstante, se requiere en ambas sociedades un porcentaje distinto de desembolso de capital social para poder constituir la sociedad.

En el caso de las SAT deberán inscribirse en el registro general de las SAT y requiere de un desembolso inicial de al menos el $25 \%$ del capital social y en el caso de las cooperativas agrarias se registrarán en el registro de cooperativas y el capital social deberá estar totalmente desembolsado.

\subsection{Número, tipos y limitaciones de los socios}

Nos centramos en este momento en la figura de los socios, así como su tipología y las limitaciones que se establecen a la condición de socios.

2. De acuerdo con el artículo 10 LC 27/1999, la escritura pública de constitución de la sociedad será otorgada por todos los promotores y en ella se expresará:

a) La identidad de los otorgantes.

b) Manifestación de éstos de que reúnen los requisitos necesarios para ser socios.

c) La voluntad de constituir una sociedad cooperativa y la clase de que se trate,

d) Acreditación por los otorgantes de haber suscrito la aportación obligatoria mínima al capital social para ser socio, y de haberla desembolsado, al menos, en la proporción exigida estatutariamente.

e) Si las hubiere, valor asignado a las aportaciones no dinerarias, haciendo constar sus datos registrales, si existieren, con detalle de las realizadas por los distintos promotores.

f) Acreditación de los otorgantes de que el importe total de las aportaciones desembolsadas no es inferior al capital social mínimo establecido estatutariamente.

g) Identificación de las personas que, una vez inscrita la sociedad, han de ocupar los distintos cargos del primer Consejo Rector, el de interventor o interventores y declaración de que no están incursos en causa de incapacidad o prohibición alguna para desempeñarlos establecida en esta u otra Ley.

h) Declaración de que no existe otra entidad con idéntica denominación, a cuyo efecto se presentará al notario la oportuna certificación acreditativa por el Registro de Sociedades Cooperativas.

i) Los Estatutos. 
Respecto al número de socios necesarios para poder constituirse en una SAT debe haber como mínimo 3 que podrán ser: bien personas físicas que tengan la condición de titular de la explotación agraria o trabajadores agrícolas; o bien personas jurídicas cuyo objetivo sea la realización de fines agrarios, aunque no tengan la condición de titular de una explotación agraria ni la consideración de trabajadores agrícolas. En este caso, dichas personas jurídicas deberán otorgar apoderamiento suficiente a sus representantes que les faculte y habilite para intervenir como tales.

Es importe poner de manifiesto las limitaciones que se establecen a las aportaciones de los socios en las SAT. En primer término, en el caso de socios personas físicas un socio no puede tener más de la tercera parte del capital. Y en el caso de socios personas jurídicas el montante total de las aportaciones realizadas por el conjunto de ellos, no puede alcanzar en ningún caso el $50 \%$ del capital social (artículo 8.5 RD 1776/81).

Además, ningún socio podrá adquirir productos elaborados por la SAT con ánimo de lucrarse en la reventa. El objetivo de tal prohibición es que ninguno de los socios ejerza su actividad a título individual, sino que sea una unión de socios con fines comunes

Conviene en este momento, detenerse en la figura del socio de una cooperativa agraria puesto que hay un espectro mucho más amplio que en las SAT. En primer lugar, en cuanto al número de socios necesarios para constituir las cooperativas, la legislación hace una distinción según se trate de cooperativas de primer y segundo grado. En las cooperativas de primer grado exige un mínimo de tres socios y en las de segundo grado al menos dos empresas cooperativas.

Y respecto al tipo de socios de las cooperativas en general, podrán ser tanto personas físicas como jurídicas, públicas o privadas, así como, comunidades de bienes. Además, se permite a los Estatutos la posibilidad de establecer unos requisitos necesarios para la adquisición de la condición de socio, en virtud del artículo 12 de la Ley de Cooperativas.

$\mathrm{Al}$ respecto, se considera que pueden existir los siguientes cuatro tipos de socios en las cooperativas de carácter general siguiendo a Tomillo (1997:109):

En primer lugar, los socios cooperativistas típicos que cumplen con un doble compromiso de permanencia en la sociedad porque están comprometidos en la actividad cooperativa por un lado y por otro en su financiación (Macías Ruano, 2021:222230). No obstante, no son trabajadores de esta, sino que son titulares de una empresa cuyo objetivo consiste en obtener bienes y servicios operando juntamente con los demás usuarios de la cooperativa para reducir costes y por tanto colaborando con la financiación de la actividad. 
En segundo lugar, los socios colaboradores o asociados. En particular, el artículo 14 de la LC 27/1999 los define como "personas físicas o jurídicas, que, sin poder desarrollar o participar en la actividad cooperativizada propia del objeto social de la cooperativa, pueden contribuir a su consecución”.

En efecto, únicamente tienen un único compromiso, la financiación de la actividad cooperativa y además se les exige un desembolso económico que determine la Asamblea General, sin exigirle nuevas aportaciones de capital. Las aportaciones realizadas por este tipo de socios en ningún caso podrán exceder del cuarenta y cinco por ciento del total de las aportaciones al capital social, ni el conjunto de los votos a ellos correspondientes, sumados entre sí, podrán superar el treinta por ciento de los votos en los órganos sociales de la cooperativa.

En tercer lugar, el socio trabajador que es el socio de la sociedad cooperativa de trabajo asociado y explotación comunitaria de la tierra. Se trata de un socio, que "cooperativiza" su trabajo y colabora económicamente en proporcionar los recursos financieros a la sociedad cooperativa en la que se integra en un puesto de trabajo.

Por tanto, la diferencia con el socio típico se basa en que los socios trabajadores trabajan conjuntamente para producir bienes y servicios que van a adquirirse en el mercado por personas ajenas a la sociedad cooperativa.

Y por último, el socio de trabajo que se trata de una figura equivalente a la del socio trabajador en las cooperativas de segundo grado y en las cooperativas de primer grado, que no sean trabajo asociado ni explotación comunitaria. No obstante, los Estatutos podrán prever la admisión de este tipo de socios, cuya actividad cooperativizada consistirá en la prestación de su trabajo personal en la cooperativa (art 13.4 LC 27/1999).

Además, en el caso particular de las cooperativas agrarias podrán considerarse socios de pleno derecho como personas físicas: los titulares de explotaciones agrícolas, ganaderas o forestales; incluyendo a las personas titulares de estas explotaciones en régimen de titularidad compartida. Y como personas jurídicas: las SAT; las comunidades de regantes; las comunidades de aguas; las comunidades de bienes y las sociedades civiles o mercantiles que tengan el mismo objeto social o actividad complementaria enumerada en la definición de cooperativa agraria establecida anteriormente. (art 93 LC 27/1999).

$\mathrm{Y}$ en cuanto a las limitaciones que se establecen a las aportaciones de socios en las cooperativas en general y en las cooperativas agroalimentarias en particular, no se hacen distinciones entre los socios personas físicas y jurídicas, como si ocurre en las SAT, sino que son las siguientes: 
- En el caso de cooperativas de primer grado el importe total de las aportaciones de cada socio no podrá exceder de un tercio del capital social;

- En el caso de socios cooperativas sin ánimo de lucro o sociedades participadas mayoritariamente por cooperativas, se estará a lo dispuesto en los estatutos o asamblea general;

- Y cuando las aportaciones son realizadas por los socios colaboradores en ningún caso podrán exceder del cuarenta y cinco por ciento del total de las aportaciones al capital social.

Por tanto, mientras el número mínimo de socios para constituir la SAT será de 3 que pueden ser tantas personas físicas como jurídicas, pero con unas limitaciones a sus aportaciones al capital social, en las cooperativas agrarias serán 3 o 2 socios mínimos según se trate de cooperativas de primer o segundo grado y cuya tipología de socios es mucho amplia que en las SAT, incluyendo además de titulares de explotaciones agrarias, ganaderas y forestales, a las propias SAT y comunidades de bienes entre otros.

\subsection{Procedimiento de adquisición, transmisión y pérdida de la condición de socios}

A continuación, vamos a estudiar el proceso de adquisición, transmisión y pérdida de la condición de socio tanto en la SAT como en la cooperativa agraria.

En principio, el Real Decreto 1776/81 que regula las SAT deja dicha regulación a los Estatutos de la sociedad. En particular, según el artículo 6 del Real Decreto 1776/81; "Los Estatutos sociales regularán necesariamente las condiciones de ingreso de los socios, así como las causas de baja y sus efectos".

No obstante, es importante recalcar que la legislación establece unas limitaciones en cuanto a las aportaciones máximas de cada socio al capital social según se trate de socios personas físicas o jurídicas y que hemos analizado en el apartado anterior.

El RD 1776/81 permite la posibilidad de adquirir la condición de socio por transmisión de los resguardos nominativos representativos del capital, puesto que los Estatutos sociales deberán expresar "los efectos de la transmisión de las aportaciones sociales por actos inter vivos o mortis causa, salvaguardando el derecho de continuidad de los herederos como socios si estos reúnen las condiciones exigidas en los Artículos 5 y 6 del RD 1776/81".

En efecto, de acuerdo con los artículos 5 y 12 RD 1776/81 está permitida la transmisión en operaciones intervivos, dentro de las condiciones fijadas en los estatutos, al igual que se admiten las trasmisiones mortis causa, salvaguardando así los derechos de los herederos. 
Por tanto, la SAT se trata de una sociedad abierta en el que es posible la entrada y salida de socios libremente sin que tenga que ser necesario tener la condición de socios previamente.

En todo caso, se considerará causa de baja de la condición de socios en una SAT:

a) La transmisión total de su participación por actos inter vivos de la condición de socio.

b) La muerte o incapacidad legal del socio.

c) La separación voluntaria.

d) Y la exclusión forzosa. En este caso, la Asamblea General de la sociedad se encargará de la exclusión forzosa de algún socio, siendo necesario en este caso voto favorable de la mayoría absoluta (art. 6.2 Real Decreto 1776/81”.).

La baja del socio implicará la liquidación definitiva de su participación en el patrimonio social en la cuantía que corresponda y los Estatutos sociales habrán de establecer el régimen aplicable a cada liquidación. Además, el socio que cause baja continuará siendo responsable frente a la SAT del cumplimiento de las obligaciones contraídas hasta la fecha de pérdida de su condición de socio (art. 6.3). Aunque el artículo 12.3 e) dispone que los Estatutos establecerán "la forma y plazos de liquidación por cese como socio".

En el caso de las cooperativas agrarias, también se recoge en la legislación que se establecerán en los estatutos de la sociedad los requisitos necesarios para la adquisición de la condición de socio. No obstante, en el artículo 13 de la Ley de Cooperativas se establece explícitamente, a diferencia de las SAT, que la adquisición de la condición de socio está condicionado a suscribir la aportación obligatoria al capital social que le corresponda y efectuar su desembolso y además, cumpliendo las limitaciones que se establecen a la aportación de un solo socio al capital social analizado anteriormente.

En cuanto al procedimiento de transmisión de las aportaciones, de acuerdo con el artículo 50 de la LC 27/1999, se establecen dos posibilidades al igual que en las SAT:

- Por actos inter vivos. En este caso, a diferencia de las SAT, las aportaciones únicamente podrán transmitirse a otros socios de la cooperativa y a quienes adquieran la condición de socio dentro de los tres meses siguientes a la transmisión.

Por tanto, la voluntad del legislador en las cooperativas es limitar la transmisión de las aportaciones internamente a los socios de la cooperativa, en el momento actual o que adquieran la condición de socios en tres meses desde la transmisión, ya que si no lo hacen durante dicho plazo no podrán adquirir las aportaciones. 
- Por sucesión mortis causa a los causahabientes si fueran socios y así lo soliciten, y si no fueran socios previa admisión como tales en plazo de seis meses. Es decir, como causahabientes la legislación se refiere a los herederos o personan que recibe bienes y derechos como consecuencia del fallecimiento del causante. Se vuelve a poner de manifiesto, la necesidad de tener la condición de socios o solicitar la admisión en el plazo de seis meses como socios para que los herederos puedan adquirir las aportaciones. Con lo cual, también en este caso, está condicionada la trasmisión de las participaciones a tener la condición de socio, mientras que en las SAT solo se exige a los herederos que reúnan las características para ser socios de las SAT sin la necesidad de ser previamente socios.

Por último, procedemos a analizar la pérdida de la condición de socio cooperativo en los términos previstos por el artículo 17 de la Ley de Cooperativas.

Se considera que el término "baja" no tiene por qué suponer la pérdida de la condición de socio (ruptura de los vínculos con la sociedad cooperativa), porque en determinados casos la baja de la condición de socio típica puede ir asociada al alta como asociado. En particular, se deducen dos tipos de bajas, del análisis de la legislación.

En primer lugar, la baja voluntaria que se produce de forma unilateral por propia voluntad del socio, y en cualquier momento, mediante preaviso por escrito al Consejo Rector de la Cooperativa. En este sentido, el plazo de preaviso será el que se fije en los Estatutos, y en ningún caso superior a un año. Conviene resaltar, que su incumplimiento podrá dar lugar a indemnización de daños y perjuicios.

A su vez, distinguimos tres tipos de bajas voluntarias:

- Baja voluntaria justificada de orden general (art 17.3 LC 27/1999). En este supuesto, los Estatutos podrán exigir el compromiso del socio de no darse de baja voluntariamente sin una causa justificada hasta el final del ejercicio económico en el que desea causar baja o hasta que haya transcurrido desde su admisión el tiempo que fijen en los Estatutos, que no será superior a cinco años.

- Baja voluntaria justificada con justificación legal explicita (art. 17.4 LC 27/1999). En el caso del socio que hubiese salvado expresamente su voto o estuviese ausente y disconforme con cualquier acuerdo de la Asamblea General, que implique la asunción de obligaciones o cargas gravemente onerosas no previstas en los Estatutos, podrá darse de baja, y tendrá la consideración de justificada, mediante escrito dirigido al Consejo Rector dentro de los cuarenta días a contar del siguiente al de la recepción del acuerdo.

- Y la baja voluntaria injustificada se produce cuando la causa no se considere justificada por los Estatutos o no se considere justa por el Consejo Rector, la Asamblea, Juez o tribunal. 
En segundo lugar, la baja forzosa que se produce por la pérdida de los requisitos necesarios para la consideración de socio, en virtud de lo que establece la Ley o los Estatutos de la Cooperativa. En particular, se acordará previa audiencia del interesado por el Consejo Rector, de oficio, a petición de cualquier otro socio o del propio afectado (art 17.5 LC 27/1999).

Además de la baja, ya sea voluntaria o forzosa, existe otro caso en el que se puede perder la condición de socio cooperativista. Se trata, del proceso de expulsión en el caso de la realización por parte del socio de una conducta reprochable muy grave en su relación societaria. Respecto al procedimiento sancionador, se establecerá en los Estatutos y será ejecutivo una vez sea notificada la ratificación del Comité de Recursos o en su defecto, de la Asamblea General, mediante votación secreta; o cuando haya transcurrido el plazo para recurrir ante los mismos sin haberlo hecho.

Podemos suponer que la expulsión de un socio de una cooperativa agraria se equipara con la exclusión forzosa de un socio de las SAT, aunque en el caso de las cooperativas queda mucho más definido y claro que se trata de una expulsión que en el caso de las SAT, que no concreta si se trata de una conducta reprochable del socio que sería equiparable a la expulsión o que simplemente el socio ha perdido los requisitos para la consideración de socio de las SAT y por eso debe perder la condición de socio y que sería equiparable a la baja forzosa.

En efecto, tanto en las SAT como en las cooperativas el procedimiento de adquisición, transmisión y baja de la condición de socios viene determinado por lo que se establece en los estatutos siempre respetando las limitaciones a las aportaciones máximas de los socios. Sin embargo, las SAT son sociedades muchas más abiertas y menos restrictivas que las cooperativas en lo que se refiere al procedimiento de transmisión, ya que no está limitado a tener la condición de socios previamente ni es necesario adquirirla en un plazo determinado como ocurre en la cooperativa agraria.

\subsection{Responsabilidad de los socios y reparto de beneficios de la sociedad}

En cuanto el tipo de responsabilidad de los socios, en la SAT sigue las mismas pautas que la sociedad civil, siempre y cuando no se haya pactado otra cosa en los Estatutos de la sociedad. Por tanto, si no se pacta otro tipo de responsabilidad, según el artículo 1.2 del Real Decreto 1776/81, “de las deudas sociales responderá en primer lugar el patrimonio social, y subsidiariamente el patrimonio de los socios, de forma mancomunada e ilimitada, salvo que los estatutos hubieran establecido otra cosa”.

En resumen, la responsabilidad de los socios en una SAT es por un lado; ilimitada puesto que el socio responde frente a terceros con su patrimonio, que es 
independiente del patrimonio social; además, subsidiaria, ya que una vez liquidado el patrimonio de la sociedad, si éste no alcanza para cubrir las deudas, responden los socios con sus bienes presentes y futuros; y por último, mancomunada, con lo que la obligación se fragmenta en tantas deudas como socios (art. 1138 del Código Civil). El socio responde "por su parte" y no "por el todo".

Sin embargo, la responsabilidad de los socios en una cooperativa agraria se limita a las aportaciones del capital social que hubieran suscrito, estén o no desembolsadas en su totalidad (art 15.3 LC 27/1999).

Por tanto, se trata de una responsabilidad de los socios limitada al capital social y por tanto responden de las deudas de la sociedad frente a terceros limitada a su aportación, al contrario que las SAT.

Centrándonos en el criterio de reparto de beneficios de la sociedad, cabe destacar que en las SAT se sigue el criterio general capitalista de reparto de beneficios en función de la aportación del capital de los socios como establece Mauleón (2015: 29-34) y así desde mi punto de vista se desprende del artículo 7 del RD 1776/1981 que regula las SAT y que textualmente establece que "las ganancias o beneficios comunes serán proporcionales a su participación”.

No obstante, es importante hacer una puntualización a este aspecto, ya que la doctrina considera que la voluntad del legislador es ambigua porque no se sabe si se refiere expresamente a su participación en el capital social o bien en la actividad que realiza el socio en la entidad. Por tanto, hay quienes consideran que la norma pretende el reparto de beneficios proporcional al capital social, mientras que quienes consideran que el reparto de beneficios es proporcional a la participación del socio en la actividad de las SAT.

Respecto a las cooperativas agrarias el criterio del legislador es muy claro y el reparto de beneficios será proporcional a la actividad del socio en la cooperativa. Concretarme el término fiscal se denomina "Retorno cooperativo"3 y se define como la cuantía de excedente neto del ejercicio económico que queda una vez deducidas las dotaciones de fondos obligatorios y que se reparte entre los socios en proporción a las operaciones, actividades o servicios cooperativizados realizados por cado socio en la cooperativa. 


\subsection{Extinción de la sociedad}

Vamos a referirnos a continuación a los procesos por los que se extingue la SAT y la cooperativa agraria y que en ambos casos son el proceso de disolución y de liquidación.

En virtud del artículo 13.1 del RD 1776/81 y 70 de la LC 27/1999 se enumeran las siguientes causas de disolución de ambas sociedades, que son básicamente similares, pero con relevantes matices:

- Por el acuerdo de disolución por parte de la Asamblea General, que deberá ser adoptado en el caso de las SAT en primera convocatoria por dos tercios de los socios y en segunda convocatoria por mayoría simple, debiendo estar en todo caso representado al menos el 50\% del capital. Cabe destacar que este acuerdo de disolución se trata de un acuerdo extraordinario en el que no se establecen las mismas mayorías legales o estatutarias exigidas con carácter general para la adopción de acuerdos. Sin embargo, en el caso de las cooperativas no se distingue entre primera convocatoria ni segunda convocatoria y exige en todo caso una mayoría de dos tercios de los socios presentes y representados para adoptar el acuerdo de disolución de la sociedad.

- El Cumplimiento del plazo para el que se habían constituido fijado en los estatutos, salvo que se hubiera acordado su continuación con anterioridad.

- La conclusión del objeto social o imposibilidad de realizarlo. En realidad, se trata de dos causas como son; el agotamiento del objeto social, en caso de finalizar un proyecto o bien cuando dicha sociedad no pueda realizarlo por cualquier causa.

- La cesación o abandono de actividades sociales durante un periodo continuado de 2 años. Es decir, la inactividad de la sociedad y el cambio del objeto social de la misma. En el caso de las cooperativas, incluye además la paralización de la actividad de los órganos sociales durante al menos dos años.

- En el caso de las SAT, la alteración sustancial de los caracteres propios que configuran la SAT constituye una causa de disolución porque dejan de cumplirse los requisitos que determinaron su inscripción. Con dicha causa de disolución, se pone de manifiesto el carácter de sociedad controlada por los poderes públicos, configurándose la disolución como una sanción.

- En el caso de las cooperativas se incluyen dos causas adicionales que no se contemplan en las SAT, y que son en primer lugar, por fusión, absorción o escisión total de la sociedad y, en segundo lugar, la reducción del número de socios por debajo del capital social mínimo establecido en los estatutos sin que se haya reestablecido en el plazo de un año. 
Conviene destacar, que cualquier causa de disolución de la SAT deberá inscribirse en el Registro Oficial de SAT y publicarse en el Registro Oficial del Estado (art. 13.3 del RD 1776/81). En caso de que concurra una causa de disolución y no sea acordada por la Asamblea General, el Ministerio de Agricultura o el Registro de SAT de cada Comunidad Autónoma o cualquiera de los socios podrá solicitar al órgano competente del orden de la jurisdicción civil que declare disuelta la sociedad (art. 13.2 del RD 1776/81).

En este sentido, cuando se trate de una cooperativa agraria el acuerdo de disolución deberá elevarse a escritura pública o en su caso la resolución judicial o administrativa se inscribirá en el Registro de Sociedades Cooperativas y deberá publicarse en uno de los diarios de mayor circulación de la provincia del domicilio social (art. 70 Ley de cooperativas).

Con la disolución se inicia el proceso de liquidación. Por tanto, el proceso de liquidación constituye la última fase de la vida social.

En el caso de las SAT y durante el periodo de liquidación, la SAT conservará su personalidad jurídica, debiendo añadir a su nombre y número la frase "en liquidación" (art.14.1 RD 1776/81).

La duración de dicha fase será de un año y rebasado dicho año podrá realizarse de oficio la cancelación de la SAT en el Registro General (art. 14.2 RD 1776/81). Dicha cancelación, determinará la perdida de la condición y los beneficios de la SAT, pero no la destrucción del contrato de sociedad y de la sociedad misma, que seguirá viva en tanto mantenga relaciones jurídicas.

No obstante, dicha limitación a un año de la fase de liquidación no tiene sentido cuando la sociedad tiene contratos pendientes de ejecución de una duración mayor.

En el caso de las cooperativas agrarias no se establece ninguna limitación de la duración de la fase de liquidación.

Respecto a los liquidadores, en las SAT serán elegidos por la asamblea general y serán un número impar de socios no superior a cinco. Si no fuera posible, la formarán los miembros de la Junta Rectora en la fecha de disolución y, asimismo, actuará colegiadamente y sus acuerdos se transcribirán en el libro de actas. En este sentido. parece deducirse que tanto el presidente como la Junta Rectora cesarán en su cargo y serán sustituidos por la Comisión de Liquidación (artículo 14.3 RD 1776/81).

Los liquidadores de las cooperativas se fijarán en los estatutos de la sociedad, y en el caso de que no se hay previsto, la Asamblea General designará entre los socios en votación secreta y por mayoría de votos a los liquidadores que deberán ser un número impar al igual que en las SAT, sin embargo, no se estable límite superior de liquidadores como en las SAT. 
En el plazo de dos meses desde la disolución, sin que se hubiese efectuado el nombramiento de liquidadores, el Consejo Rector de la cooperativa o cualquier socio podrá solicitar del Juez de Primera Instancia su designación, que podrá recaer en personas no socios efectuándose el nombramiento en el plazo de un mes.

Hasta el nombramiento de los liquidadores, el Consejo Rector continuará en las funciones gestoras y representativas de la sociedad.

Una vez aprobado la disolución, se procederá a solicitar en el caso de las SAT la cancelación en el Registro General de SAT y se publicará en el BOE y en el BOP donde radique su domicilio social. En el caso de las cooperativas, finalizada la liquidación se otorgará en escritura pública la extinción de la sociedad y dicha escritura se inscribirá en el Registro de sociedades cooperativas.

\section{7. Órganos de gestión}

En cuanto a los órganos de gestión, tanto las SAT como las cooperativas agrarias se componen de tres órganos de gestión. Sin embargo, en el caso de las cooperativas, la estructura y organización es mucho más compleja como veremos a continuación.

Empezamos por la estructura de gestión de las SAT que se compone por tres órganos (art. 10). El primero de ellos es la Asamblea General, un órgano supremo de expresión de la voluntad de los socios y constituida por todos ellos. El Real Decreto de las SAT concede gran importancia a la regulación estatutaria de este órgano.

En particular, el artículo 12.3h RD 1776/81) establece que los Estatutos sociales expresarán el quórum requerido de personas o de capital para la toma de acuerdos en la Asamblea General y la expresión concreta de cuáles son estos según materias.

Por lo que se refiere a las mayorías exigidas para adoptar acuerdos, el artículo 11.1 RD 1776/81 establece que los acuerdos de la Asamblea General salvo disposición contraria de los Estatutos se adoptaran por mayoría simple de los asistentes. Sin embargo, a pesar de que todos los socios se someten a los acuerdos de la Asamblea General, existirá la facultad de impugnarlos en los casos que determine el Real Decreto, siempre y cuando los socios que hubiesen asistido a dicha junta hubiesen hecho constar en Acta su oposición al acuerdo impugnado, y los que hayan sido privados ilegítimamente de emitir su voto.

El segundo órgano de las SAT se considera la Junta Rectora. Constituye un órgano de gobierno, representación y administración ordinaria de la SAT. No obstante, si el número de socios es inferior a diez, la Asamblea General asumirá como propias las funciones que competen a la Junta Rectora, constituyendo un solo órgano que estará formado por un presidente; un secretario; tres vocales como mínimo y doce como máximo; siempre que tengan la condición de socios. 
La elección de esta corresponde exclusivamente a la Asamblea General. Por otro lado, la Junta Rectora solo se reunirá válidamente con la presencia de la mitad de sus miembros y adoptará sus decisiones por mayoría simple de asistentes (art .11.1 RD 1776/81). En caso de empate, el presidente dirimirá con su voto los empates en la votación de uno y otro órgano social. Además, sus acuerdos pueden ser impugnados en los mismos términos que se pueden impugnar los acuerdos de la Asamblea General.

Y, por último, el presidente, órgano unipersonal con las facultades estatutarias que incluirán necesariamente la representación de la SAT sin perjuicio de las conferidas a la Junta Rectora.

En el caso de las cooperativas y de acuerdo con el artículo 19 de la LC 27/1999 son tres órganos de gestión:

En primer lugar, la Asamblea General tiene como objeto deliberar y adoptar acuerdos sobre aquellos asuntos que legal o estatutariamente sean de su competencia, vinculando las decisiones adoptadas a todos los socios de la cooperativa. Con carácter general, en este órgano se rige el principio de un socio un voto, (Cañabate Pozo, 2020:230) pero existen excepciones a este principio y tiene mayores competencias que las juntas de las sociedades mercantiles capitalistas.

El segundo órgano, es el Consejo Rector el órgano colegiado de gobierno al que corresponde, al menos, la alta gestión, la supervisión de los directivos y la representación de la sociedad cooperativa, con sujeción a la Ley, a los Estatutos y a la política general fijada por la Asamblea General. Cabe destacar, que este órgano solo puede ser sustituido por un administrador único en las cooperativas de menos de diez socios.

Por último, la Intervención tiene como objetivo un papel fiscalizador de las cooperativas pudiendo consultar y comprobar toda la documentación de la cooperativa y proceder a las verificaciones que estime necesarias. Se puede considera que a este órgano solo se le pueden atribuir funciones reales en el marco de la gestión de una gran cooperativa, ya que en las pequeñas cooperativas debería prescindirse de su obligatoriedad para no agravar su Estatuto respecto al de otras sociedades de parecida tipología. Los Estatutos podrán regular un límite de votos que ostenten los socios en relación con el conjunto de votos sociales de la cooperativa. Pues, se plantea la posibilidad de un voto plural ponderado, en proporción al volumen de la actividad cooperativizada del socio, que no podrá ser superior en ningún caso a cinco votos sociales sin que puedan atribuir a un solo socio más de un tercio de los votos totales de la cooperativa.

Podemos afirmar con rotundidad que en cuanto al régimen, control y organización se considera en las cooperativas en general y en las agrarias en particular, mucho más estricto que en otro tipo de sociedades agrarias como las SAT porque impone una Intervención como órgano de control. 
(pp. 165-197)

\subsection{Fiscalidad}

En primer lugar, es importante volver a poner de manifiesto la especial protección que otorgan los poderes públicos a las SAT y a las cooperativas agrarias en materia fiscal, por constituir la principal forma de asociacionismo en el sector agrario y por considerar al sector agrario en virtud, del artículo 130 de la Constitución Española, un sector que requiere una atención especial por parte de la administración.

Las principales diferencias entre las SAT y las cooperativas agrarias en materia tributaria hacen referencia a la tributación directa y en particular, los impuestos sobre bienes inmuebles e impuesto sobre sociedades (Aguilar Rubio, 2020:262).

Respecto al impuesto sobre bienes inmuebles, que como su nombre indica es un impuesto que grava la titularidad dominial y otros derechos reales que recaen sobre bienes inmuebles, las cooperativas agrarias a diferencia de las SAT disfrutan de una bonificación especial del 95 por ciento de la cuota y de los recargos de los bienes de naturaleza rústica de las cooperativas agrarias, como establece el artículo 33 de la Ley 20/1990 sobre el Régimen fiscal de las cooperativas, en adelante LRFC.

Nos detenemos en este momento en el análisis de la regulación de la tributación del impuesto de sociedades en las SAT y las cooperativas agrarias.

En el caso de las SAT tributan al régimen general siguiendo los criterios establecen en la Ley 27/2014 del Impuesto sobre Sociedades que regula en España la fiscalidad directa en el ámbito de la actividad económica desarrollada por personas jurídicas.

En particular las SAT tributarán al tipo impositivo general del $25 \%$.

Cabe destacar que es la propia Ley 27/2014 del Impuesto sobre Sociedades la que establece que permanecerán al margen de esta ley determinadas normas específicas como es el caso de la LRFC.

Por tanto, lo que se deduce del preámbulo de esta ley es que se mantiene un régimen fiscal especial para las cooperativas en general y las cooperativas agrarias en particular.

En efecto, la LC 20/1990 distingue entre cooperativas protegidas y especialmente protegidas. Por su parte se incluyen este segundo grupo de cooperativas especialmente protegidas las cooperativas agrarias.

Con carácter general, en las cooperativas se establecerán un tipo impositivo de tributación de del 20 por ciento para los resultados cooperativos (art. 33.2 LRFC) que se definen como aquellos resultados que derivan de las operaciones cooperativas con los socios. No obstante, si se trata de resultados extracooperativos derivados de la actividad cooperativizada con terceros, la LRFC establece la misma tributación que el resto de las personas jurídicas, es decir se aplicará un tipo impositivo general del 25 por ciento. 
Y además de disfrutar de una rebaja en el tipo impositivo, en el impuesto de sociedades, las cooperativas agrarias por ser cooperativas especialmente protegidas disfrutarán de una bonificación del 50 por ciento de la cuota íntegra que constituye la cantidad resultante de aplicar a las bases imponibles positivas o negativas los tipos de gravamen.

Con lo cual, queda constatada la protección especial en material del impuesto de sociedades que se otorgan a las cooperativas agrarias por partida doble, porque además de aplicar un tipo impositivo inferior al resto de sociedades entre las que se incluye las SAT, se aplica una bonificación del 50 por ciento a la cuota íntegra.

\section{Conclusiones}

La empresa agraria se distingue de la empresa comercial básicamente por el requisito de profesionalidad que se le exige al titular de la explotación, el empresario agrario, a diferencia del titular de la empresa comercial, "el comerciante". Se le exige dicha profesionalidad porque el desarrollo de la actividad agraria representa una política de estado por su función social, por ello está sometida a una serie de estímulos y subvenciones en el marco de la Política Agraria Comunitaria y por tanto la profesionalidad se considera necesaria para poder optar a fondos públicos.

Desde el punto de vista del sujeto titular de la empresa agraria, la empresa agraria se distingue en base a dos criterios. El primero de ellos, hace referencia a la estructura jurídica de la empresa. En base a este criterio, tanto la SAT como las Cooperativas agrarias se incluyen dentro de la categoría de empresas agrarias asociativas. Por tanto, tienen personalidad jurídica independientemente de sus socios y su objeto social se vincula a la actividad agraria.

Cabe destacar que tanto la SAT como la Cooperativa agraria constituyen la forma tradicional y ampliamente aceptada de asociacionismo agrario, por tanto, el interés del presente artículo radica a la importancia de realizar una comparativa legislativa entre ambas sociedades con el objetivo de ser relevante para la elección de la forma jurídica idónea del empresario agrario.

Después del análisis realizado en este artículo, conviene concluir desgranando una serie de diferencias.

En primer lugar, cabe destacar que mientras la SAT se considera sociedad de carácter civil porque así lo define el propio RD 1776/81, la cooperativa agraria tiene una finalidad mercantil "especial" por su estructura compleja de gestión a diferencia de las sociedades propiamente mercantiles. 
Respecto el objeto social, en las SAT se considera mucho más amplio, pues se incluye la producción, comercialización y transformación de productos agrarios, ganaderos o forestales, entre otros, mientras que en las cooperativas agrarias la función principal es la de comercialización.

En cuanto a la constitución, tanto la SAT como las cooperativas agrarias deberán inscribirse de forma obligatoria en sus registros especiales para adquirir la personalidad jurídica, concretamente en el Registro general de las SAT y el registro de cooperativa. No obstante, se requiere en ambas sociedades un porcentaje distinto de desembolso de capital social para poder constituir la sociedad.

En el caso de las SAT se requiere de un desembolso inicial de al menos el $25 \%$ del capital social y en el caso de las cooperativas agrarias, el importe total de las aportaciones desembolsadas no puede ser inferior al del capital social mínimo establecido estatutariamente, lo que significa que debe estar totalmente desembolsado.

Tanto en las SAT como las cooperativas agrarias pueden ostentar la condición de socios personas físicas como personas jurídicas. En el caso de socios personas físicas podrán ser titulares de explotaciones agrarias, pero además en las SAT trabajadores agrarios. Sin embargo, en el caso de socios personas jurídicas en las cooperativas agrarias se incluyen además de personas jurídicas cuyo objeto social tenga fines agrarios, las propias SAT, y comunidades de bienes. Y, además, se establece una limitación máxima de aportación por un solo socio que en el caso de las SAT es distinto si es persona física o jurídica y en el caso de las cooperativas agrarias es el mismo límite en las cooperativas de primer grado sean o no personas jurídicas.

En cuanto al procedimiento de adquisición, transmisión y baja de la condición de socios tanto en las SAT como en las cooperativas, viene determinado por lo que se establece en los estatutos. Sin embargo, las SAT son sociedades muchas más abiertas y menos restrictivas que las cooperativas en lo que se refiere al procedimiento de transmisión, ya que no está limitado a tener la condición de socios previamente ni es necesario adquirirla en un plazo determinado como ocurre en la cooperativa agraria.

Centrándonos en la responsabilidad y reparto de beneficios de los socios, mientras que en las SAT los socios responden frente a terceros de forma ilimitada con su patrimonio y el reparto de los beneficios es proporcional a la aportación al capital de cada socio o según los estatutos, en las cooperativas la responsabilidad frente a las deudas sociales se limita a la aportación al capital social y el reparto de beneficios es proporcional a las operaciones, actividades o servicios que desarrollen los socios en la cooperativa.

El proceso de disolución y liquidación de la sociedad está mucho más definido y detallado en las SAT que en las cooperativas, ya que una vez aprobada la disolución de la sociedad se inicia en ambas sociedades la fase de liquidación de la sociedad 
que estará limitada, en el caso de las SAT a un año y con unos liquidadores que deberán ser un número impar, pero con un límite máximo de cinco, mientras que en las cooperativa agrarias no se especifica el plazo máximo de duración de la fase de liquidación ni el número máximo de liquidadores, aunque también tiene que ser un número impar.

Cabe destacar que en cuanto al régimen, control y organización se considera en las cooperativas en general y en las agrarias en particular es mucho más estricto que en otro tipo de sociedades agrarias como las SAT porque impone una Intervención como órgano de control y con un papel fiscalizador.

Para finalizar, en materia tributaria las SAT y las cooperativas agrarias presentan importantes diferencias en tributación directa. En particular, las cooperativas agrarias disfrutan de una bonificación especial del 95 por ciento de la cuota y de los recargos de los bienes de naturaleza rústica y tributan a un tipo impositivo especial del 20 por ciento en el impuesto de sociedades, mientras que las SAT no disfrutan de ninguna bonificación en el IBI y tributan al tipo impositivo general del $25 \%$ sobre el impuesto de sociedades. 


\section{Biblioagrafía}

AGUILAR RUBIO, M.: "Análisis crítico de la fiscalidad de las cooperativas de segundo grado como fórmula de integración”, REVESCO. Revista de estudios cooperativos, no 126, 2017, pp. 118-132. DOI: https://doi.org/10.5209/REVE.58393 AGUILAR RUBIO, M.: "El régimen tributario de las Sociedades Agrarias de Transformación”. CIRIEC-España, Revista jurídica de Economía Social y Cooperativa, no 37, 2020, pp. 247-281. DOI: https://doi.org/10.7203/CIRIEC-JUR.37.16947

AMAT LLOMBART, P.: "Estatuto jurídico y fiscal de las cooperativas agrarias y agroalimentarias españolas en el marco de las empresas de economía social”, Estudios agrarios, $\mathrm{n}^{\circ}$ 15(41), 2009, pp. 9-35.

ARROYO MARTÍNEZ, I.: Legislación sobre cooperativas, Editorial Tecnos, Madrid, 1995.

BAAMONDE NOCHE, E.: "Las Cooperativas Agrarias ante el Siglo XXI", Anuario de Estudios Cooperativos, no 1, 2000, pp. 41-50.

BALBONTÍN DEL VALLE, P.: "La mejor opción para el agricultor: cooperativas agrarias", Andalucía Económica, no 136, 2002, pp. 1-32.

BELTRÁN SÁNCHEZ, E.M.: "La agricultura de grupo: las sociedades agrarias de transformación”. En: El derecho agrario: su marco jurídico y económico (dir. ORDUÑA MORENO, F.J.), Tirant lo Blanch, València, 1997, pp. 73-103.

BLANCO MORALES, J.: "Aspectos destacados de la Ley Estatal de Cooperativas 27/1999, de 16 de julio", Revista universitaria de ciencias del trabajo, no 1, 2000, pp. 153-168.

BORJABAD GONZALO, P.: "La sociedad agraria de transformación”, Anuario de la Fundación Ciudad de Lleida, no 18, 2007, pp. 63-94.

CABALLERO LOZANO, J.M.: "El empresario agrícola como miembro de la Sociedad Agraria de Transformación”, Revista General de Legislación y Jurisprudencia, no 2, 2004, pp. 183-218.

CALDENTEY ALBERT, P.: "Cooperativas Agrarias", Agricultura: Revista agropecuaria y ganadera, no 760, 1995, pp. 940-949.

CAÑABATE POZO, R.: "Cooperativa y empresa familiar", CIRIEC-España, Revista Juridica de Economía Social y Cooperativa, no 37, 2020, pp. 207-246. DOI: https://doi.org/10.7203/CIRIEC-JUR.37.17424 
CARRASCO MONTEAGUDO, I.: "Cooperativas agrarias: necesidades de financiación y financiación ajena de las necesidades", REVESCO. Revista de Estudios Cooperativos, no 63, 1997, pp. 45-62.

CARRETERO GARCÍA, A.: Empresa agraria y profesionales de la agricultura en el derecho español y comunitario, Editorial Comares, Granada, 2003.

CARROZZA, A. \& ZELEDÓN ZELEDÓN, R.: Teoría general e institutos de Derecho Agrario, Editorial Astrea, Buenos Aires, 1990.

CUENCA ANAYA, F.: "Notas sobre el régimen fiscal de las Sociedades Agrarias de Transformación”, Revista de Estudios Agrosociales, no 124, 1983, pp. 49-64.

FERNÁNDEZ REYES, J.: "La nueva Ley de Cooperativas: análisis de las cooperativas agrarias", Revista CADE: doctrina y jurisprudencia, no 2, 2009, pp. 15-18.

GALLEGO SEVILLA, L.P. \& GARCÍA MARTÍNEZ, G.: Normativa de sociedades cooperativas, Universidad Politécnica de València (UPV), València, 2000.

HERRERA CAMPOS, R.: "Hacia un nuevo modelo de empresa agraria", Estudios Jurídicos de Derecho Agrario, no 15, 2005, pp. 67-74.

MACÍAS RUANO, A.J.: "El tardío reconocimiento del carácter mercantil de las sociedades cooperativas y su consecuencia”, Deusto Estudios Cooperativos (DEC), no 9, 2017, pp. 55-86. DOI: https://doi.org/10.18543/dec-9-2017pp55-86

MACÍAS RUANO, A.J.: "El socio de cooperativa y el de sociedad de capital, puntos de divergencia y convergencia en torno a los principios que dirigen la dinámica interna cooperativa. Libre adhesión, control democrático y participación económica del socio". CIRIEC-España, Revista jurídica de Economía Social y Cooperativa, no 38, 2021, pp. 217-260. DOI: https://doi.org/10.7203/CIRIEC-JUR.38.20786

MAULEÓN MENDEZ, E.: "La sociedad Agraria de transformación a la luz de los principios cooperativos de la ACI y de la ley de economía social", CIRIEC-España, Revista Jurídica de Economía Social y Cooperativa, no 27, 2015, pp. 459-494.

MILLÁN SALAS, F.: "Requisitos de la empresa agraria", Cuadernos de Estudios Empresariales, no 3, 1993, pp. 215-226.

MOZAS MORAL, A., BERNAL JURADO, E., FERNÁNDEZ UCLÉS, D., MEDINA VIRUEL, M.J. \& PUENTES POYATOS, R.: "Cooperativismo de segundo grado y adopción de las TIC", CIRIEC-España, Revista de Economía Pública, Social y Cooperativa, no 100, 2020, pp. 67-85.

DOI: https://doi.org/10.7203/CIRIEC-E.100.17712

PULGAR EZQUERRA, J.: "El concurso de la cooperativa agraria y de la sociedad agraria de transformación". En: Cooperativas agrarias y sociedades agrarias de transformación (dir. PULGAR EZQUERRA, J. \& coord. VARGAS VASSEROT, C.), Editorial Dykinson, Madrid, 2006, pp. 781-820. 
SÁNCHEZ HERNÁNDEZ, A.: "Las cooperativas de explotación comunitaria de la tierra", Revista de Derecho Agrario y Alimentario, no 15(34), 1999, pp. 22-31.

TOMILLO URBINA. J.L.: "La empresa agraria bajo otras formas sociales". En: El derecho Agrario: Marco jurídico y económico (dir. ORDUÑA MORENO, F.J.), Tirant lo Blanch, València, 1997, pp. 105-132.

VARGAS VASSEROT, C.: "La estructura orgánica de la sociedad cooperativa y el reto de la modernidad cooperativa", CIRIEC-España, Revista Jurídica de Economía Social y cooperativa, no20, 2009, pp. 59-82.

VARGAS VASSEROT, C.: "Las Sociedades Agrarias de Transformación: aproximación crítica a su régimen legal”, Revista de Derecho de Sociedades (RdS), no 35 , 2010, pp. 159-180.

VATTIER FUENZALIDA, C.: "Causa y tipo en las cooperativas agrarias", Revista de Derecho Privado, no 10, 1978a, pp. 989-1002.

VATTIER FUENZALIDA, C.: Concepto y tipos de empresa agraria en el derecho español, Universidad de León (ULe), León, 1978b. 